\title{
L'habitat du Néolithique ancien de Colombelles « Le Lazzaro » (Calvados)
}

Anne Hauzeur

\section{(2) OpenEdition}

\section{Journals}

Édition électronique

URL : http://journals.openedition.org/rao/3571

DOI : 10.4000/rao.3571

ISSN : $1775-3732$

Éditeur

Presses universitaires de Rennes

\section{Édition imprimée}

Date de publication : 31 décembre 2016

Pagination : $375-378$

ISBN : 978-2-7535-5683-6

ISSN : 0767-709X

\section{Référence électronique}

Anne Hauzeur, «L'habitat du Néolithique ancien de Colombelles « Le Lazzaro » (Calvados) », Revue archéologique de l'Ouest [En ligne], 33 | 2016, mis en ligne le 31 juillet 2017, consulté le 02 mars 2021. URL : http://journals.openedition.org/rao/3571; DOI : https://doi.org/10.4000/rao.3571 


\section{Analyses d'ouvrages}

Billard C., Bostyn F., Hamon C., Meunier K. (dir.), 2014 - L'habitat du Néolithique ancien de Colombelles "Le Lazzaro " (Calvados), Paris, SPF, 2014, 1 volume, 408 pages, comprenant 224 figures, 39 tableaux et 41 annexes, coll. « Mémoires de la Société préhistorique française; 58 ».

Ce Mémoire de la Société préhistorique française constitue la monographie du site d'habitat de Colombelles, exploré entre 2001 et 2005, en cinq campagnes de fouille programmée. L'ouvrage se découpe en six parties selon un schéma classique de rapport de fouille et de publication de site. Après une présentation du site et de son contexte, l'étude des différents types de structures archéologiques est abordée, suivie par les résultats des analyses des mobiliers archéologiques. La quatrième partie est consacrée aux données paléoenvironnementales, tandis que la cinquième envisage l'organisation spatiale du site. L'ouvrage se clôture par une synthèse collective reprenant les différents aspects évoqués dans la monographie.

La première partie de la publication (21 pages) s'attache en premier lieu à replacer le site dans son contexte archéologique, en abordant la néolithisation du Nord-Ouest de l'Europe et les grands courants chrono-culturels (Rubané sensu stricto, Rubané récent du Bassin parisien, Blicquy/ Villeneuve-Saint-Germain), le substrat mésolithique de la France septentrionale et la question des Céramiques de La Hoguette et du Limbourg. La néolithisation de l'Ouest de la France (p. 17 et sv.) donne un contexte régional précis et étayé par de nombreuses références bibliographique. En résumé, les auteurs s'appuient sur la chronologie classique du Néolithique de la France septentrionale, sur la périodisation céramique de l'Aisne et sur un groupe de Villeneuve-Saint-Germain apparaissant vers 4950 av. J.-C. Ce cadre général permet à $C$. Billard d'exposer les problématiques que suscite la découverte de ce site, au travers de son contexte archéologique, de son organisation spatiale, des pratiques funéraires, etc. Ce n'est qu'en fin de partie que le cadre géographique est donné et le site présenté. Les vestiges du Néolithique ancien couvrent une superficie de près d'un hectare, localisée sur une légère pente (on regrettera l'absence d'indication altimétrique de la fig. 2) en arrière-plan de la rive droite de l'Orne, entre estuaire, littoral et continent. Cette position ouvre des perspectives intéressantes en matière d'exploitation du milieu, notamment les ressources minérales : silex bathonien, hématite ordovicienne, schiste. Le site est érodé, avec une ablation estimée entre 40 et $60 \mathrm{~cm}$ (p. 27).
La deuxième partie (76 pages), consacrée aux structures archéologiques, est scindée en trois chapitres : les unités d'habitation déterminées sur la base des regroupements de fosses, les sépultures et les datations 14C. Le jeu des regroupements de fosses - souvent polylobées et complexes - mène l'auteur à proposer d'emblée un plan-masse (fig. 11) où figurent six " habitations très probables » et quatre autres " possibles " selon le modèle en ligne, type Cuiry-les-Chaudardes (RRBP, Aisne) ou Poses (VSG, Eure). Les recoupements de structures ne semblent pas considérés comme des événements diachrones indicateurs d'un phasage interne, tels par exemple le cas du groupe $\mathrm{H}$ et de l'unité d'habitation 5 (p. 47 et sv.). Quasiment toutes les fosses sont considérées comme des fosses latérales d'habitation. Les dixhuit sépultures relevées sur le site témoignent de pratiques funéraires variées, et de datation diverse, entre le Néolithique (2), l'âge du Bronze (5) et l'âge du Fer (1) pour les structures datées soit par le mobilier soit directement au radiocarbone sur ossements humains. Les auteurs s'interrogent sur la validité des datations 14C (directes sur ossements humains!), qui ne sont pas en adéquation avec le mobilier ou le rituel d'inhumation. C'est ainsi que les pratiques funéraires sont discutées en considérant les sépultures - sauf celle de l'âge du Fer - comme un "ensemble funéraire cohérent" (p. 98). Il est comparé aux traditions funéraires du monde rubané. L'ensemble fait néanmoins ressortir la diversité des pratiques funéraires, que ce soit au niveau du dépôt des individus, en général allongée, du milieu de décomposition ou des types de fosses sépulcrales, étroites ou larges. Du mobilier accompagnant (céramique, lithique, parure et ocre) est présent dans trois sépultures, deux néolithiques (sépultures 110 et 297) et une âge du Bronze (sépulture 225), tandis que pour trois autres sépultures, les objets lithiques sont jugés intrusifs. Le mauvais état de conservation général des ossements humains limite fortement les observations biologiques, les réduisant à des cas isolés. Le chapitre suivant est consacré aux datations radiométriques. Quinze dates ont été obtenues pour des fosses détritiques sur matériaux organiques carbonisés et pour des sépultures par datation directe sur ossement humain. Une sépulture est clairement contemporaine des structures d'habitat du Néolithique ancien (sépul- 
ture 110), avec des dates s'étalant entre 5200 et 5000 avant notre ère. On notera que les datations de l'âge du Bronze ont été confirmées par de nouvelles dates obtenues après utilisation de l'ultrafiltration, mais que - malgré la récurrence des résultats - les auteurs considèrent comme suspectes. La tendance est d'interpréter cet ensemble comme une série de sépultures implantées dans le village et contemporaines de celui-ci (p. 107).

La troisième partie est dévolue aux études de mobilier (158 pages). La céramique est attribuée à l'" étape finale du RRBP, voire au début du groupe de VSG » et le corpus comporte au minimum 87 individus décorés ou non, répartis dans 22 fosses et deux sépultures. L'auteure (K. Meunier) signale l'absence notable de grands vases de stockage et des formes ouvertes non décorées par rapport aux autres formes présentes, notamment les vases à inflexion. Seuls dix individus sont décorés au peigne, pivotant dans tous les cas (tabl.5). Parmi les techniques, les boutons (sic) sont majoritaires suivi par les incisions. Les motifs les plus fréquents du décor principal consistent en figures verticales ou en boutons appliqués sur la panse. Quelques fragments décorés pourraient faire partie d'un récipient de style Limbourg. Un phasage interne est pressenti mais sans pouvoir le préciser. La présence d'un vase entier clairement VSG est considéré comme un indice de transition entre le RRBP et le BVSG. L'auteure souligne les originalités stylistiques du corpus par rapport au RRBP final classique du centre du Bassin parisien, avec une composante VSG (p. 141-142). L'analyse des pâtes confirme l'originalité des tessons de style Limbourg - seul exemple dégraissé à la chamotte -, le recyclage du macro-outillage comme matériau dégraissant, l'utilisation de matières végétales et de coquilles, de même que l'emploi de limons décarbonatés locaux comme matrice.

Le mobilier lithique se caractérise par une grande diversité de matériaux siliceux de provenance régionales (formations bajociennes et bathoniennes), voire locales (galets). Les auteurs (F. Bostyn et al.) soulignent le contraste quantitatif et qualitatif des assemblages entre unités et notent un « déséquilibre " numérique entre le mobilier lithique des fosses de Colombelles et les sites RRBP de comparaison (vallée de l'Aisne), plus proche manifestement de sites VSG comme Poses (p. 153). L'industrie lithique est analysée par type de matières premières, avant de la caractériser sous l'angle typofonctionnel. L'importance numériques des burins, des pièces esquillées et des grattoirs est à relever, ainsi que le rapprochement typologique des éléments de faucille avec ceux du VSG. Seul le mobilier (armatures) associé aux défunts est analysé; celui des remplissages n'est pas abordé (p. 203 et sv.), bien que deux armatures jugées non associées soient présentées, notamment une armature mésolithique. La chaille est le matériau de prédilection pour les percuteurs et les rares outils réalisés aux dépens de cette matière s'accordent avec la production en silex. Les auteurs placent l'industrie lithique de Colombelles à la transition Rubané/BVSG, comparent les armatures avec des séries locales contenant du mobilier mésolithique et font de la latéralisation un critère chronologique. Deux lames de hache ou d'herminette, en schiste tacheté et en silex crétacé, sont attribuées au Néolithique ancien (p. 221 et suiv.). À noter qu'une lame de hache en métadolérite de type A atteste une présence du Néolithique moyen sur le site.

Le macro-outillage a été étudié par C. Hamon. Il se caractérise par l'exploitation de nombreux matériaux d'origine locale, sélectionnés en fonction de leur destination fonctionnelle. A côté des abondantes molettes en granite et des percuteurs en grès, quelques catégories font l'originalité du site de Colombelles : les marteaux-enclumes pour le concassage de matières minérales et les molettes de friction de type I destinées, selon l'auteure, à l'application de colorant sur peau animale. Les instruments de mouture et de broyage s'apparentent au monde rubané comme au VSG, tandis que les outils originaux sont spécifiques à l'exploitation des hématites locales.

Les fragments d'anneaux en schiste - tous d'origine minéralogique différente - témoigneraient de la présence ancienne de ce type de parure dès la phase finale du RRBP. Les perles plates en calcaire de la sépulture 110, étudiées par S. Bonnardin, C. Bourdillon et G. Querré, sont d'origine méridionale (bassin Vocontien ou Espagne), tout comme la perle en variscite (région de Huelva en Espagne).

La quatrième partie s'attache au traitement des données paléoenvironnementales (20 pages). Seuls les macrorestes carbonisés sont suffisamment nombreux pour en tirer des résultats. L'étude des restes carpologiques (M.-F. DietschSellami) met en exergue la culture de quatre céréales et d'une légumineuse, le pois. Des fruits sauvages étaient également récoltés, telles les noisettes, les mûres et les prunelles. La présence d'orge vêtue (Hordeum vulgare) et de blé nu (Triticum aestivum) est un indice chrono-culturel important en faveur d'une composante VSG ou, comme l'auteure le suggère, un témoignage de relations précoces avec le monde méditerranéen dès le Rubané, tout comme la perle en variscite (p. 279). Les résultats anthracologiques (N. Marcoux, D. Marguerie et D. Aoustin) livrent certaines informations sur la nature des fosses. Ainsi les fosses 22-1 et 300, en périphérie de l'emprise, sont envisagées comme des structures témoignant du traitement des céréales, très limitées en taxons ligneux, le chêne avec des Pomoïdées ou du noisetier. D'autres structures ont eu une "fonction architecturale", telles 164-3, 164-2, 380. Une dichotomie entre la partie septentrionale et méridionale du site est notée, sur la base de la taille des cernes de croissance, et une phase pionnière 
est proposée pour la partie centrale du site (p. 291), avec les structures 300 et 380 dont respectivement 2 et 18 charbons ont été mesurés (tabl. 36).

Dans la cinquième partie consacrée à l'organisation de l'habitat (36 pages), un premier bilan est dressé, en retenant l'hypothèse d'un site à occupation unique de onze unités d'habitation subparallèles, au sein duquel s'organise une nécropole, dont certaines sépultures recoupent les fosses latérales des maisons. On trouve ensuite un chapitre sur les données micromorphologiques des fosses, que l'on s'attendrait à voir figurer comme élément de discussion dans la partie dévolue aux structures. Les comblements de deux structures latérales d'une même unité d'habitation ont mis en évidence plusieurs phases de fonctionnement allant de la préparation des matériaux de construction en terre et végétaux au démantèlement de structures de terre crue, en relation avec du piétinement et des activités domestiques, notamment la mouture. Une évolution pédogénétique postnéolithique est également mise en évidence.

Les données quantitatives et qualitatives des mobiliers archéologiques ont été cartographiées, donnant lieu à 24 cartes de répartition spatiale (fig. 199 et suivantes). Les remontages instaurent des liens entre les fosses latérales de deux unités d'habitation (U5 et U9), ainsi que des liens de contemporanéité entre les unités 5 et 6 et les unités 3, 7 et 9 . Les auteurs concluent à un village en une rangée de maisons alignées, sans pouvoir préciser le nombre de maisons fonctionnant simultanément (p. 307). Que ce soit la répartition spatiale de la terre cuite architecturale ou celle de la céramique, ce sont les unités 5, 9, 4 et 7 qui se distinguent des autres où ces mobiliers sont nettement plus confidentiels. L'industrie lithique en silex en en chaille se répartit de façon homogène selon les auteurs, les seules différences entre unités d'habitation étant du ressort de l'assemblage qualitatif, mettant en exergue l'affiliation RRBP. La répartition spatiale du macro-outillage reflète est le reflet d'activités spécifiques de certaines unités d'habitation, parfois non alimentaires comme le polissage en zone sud (p. 325 et fig. 217). Au terme de ces études, l'antériorité des unités 2, 7 et 9 est évoquée, bien que l'accent soit mis sur « la grande homogénéité du site [à] occupation courte " (p. 326).

La dernière et sixième partie est une synthèse générale (11 pages) replaçant le site de Colombelles - " Le Lazarro » dans un contexte chronologique et culturel large. Le village est constitué d'une rangée unique de dix maisons, tels d'autres exemples du Bassin parisien, voire des exemples plus lointains comme Elsloo (NL) ou Frimmersdorf (DE). Les auteurs notent la présence $\mathrm{d}^{\text {' }}$ indices tenus de diachronie " comme les variations des assemblages lithiques dans les fosses. L'occupation est courte, sans affirmer qu'il y ait eu plusieurs phases de construction, bien que la durée du village, telle que retenue par les auteurs, ne dépasse pas une phase stylistique de la céramique. Les datations $14 \mathrm{C}$ sont à nouveau discutées pour constater que les dates sont contemporaines de sites proches ou régionaux comme Demouville (Calvados; Rubané) ou Betton (Ille-et-Vilaine; BVSG) et plus anciennes que le laps de temps généralement admis pour le RRBP, à l'aune du Ve millénaire. Colombelles est considéré comme un site pleinement rubané, dont l'origine est à rechercher du côté de la vallée de l'Aisne, mâtiné de quelques éléments originaux (variscite, orge nue, blé tendre). Le site du "Lazarro » serait aussi l'illustration d'un transfert technique du Néolithique vers le Mésolithique final par l'intermédiaire des armatures (p. 341). Pour finir, un scénario de la néolithisation régionale est proposé : des contacts à longue distance établis par les porteurs de la Céramique de La Hoguette en préalable à une " colonisation agricole ", motivée par la présence d'hématite oolithique.

Cette monographie de site est incontestablement un ouvrage de référence au niveau des données accessibles pour le Néolithique ancien le plus occidental. Malgré les regrets suscités par une érosion importante du site de Colombelles - «Le Lazarro ", les vestiges conservés et le mobilier récolté sont riches d'informations, pour le Néolithique ancien en général et pour le Néolithique normand en particulier, sans oublier la Protohistoire. Par contre le postulat de départ d'un village à occupation unique datant de la transition RRBP final/BVSG, avec de surcroît des sépultures en son sein, conditionne et contraint toutes les études à s'accorder finalement avec ce parti pris, une conviction ou un malaise que l'on perçoit comme un fil rouge tout au long de la lecture de l'ouvrage. L'application de la méthode hypothético-déductive, dont on connaît parfaitement les écueils, conduit les auteurs à écarter les évidences données par les dates 14C, notamment d'un site multipériode ou encore la mise en perspective dynamique avec des composantes nettement VSG que l'on évacue au nom d'une évolution linéaire, et à forcer les interprétations pour faire rentrer les résultats dans le tiroir ad hoc. L'exemple le plus frappant étant les dates de l'âge du Bronze obtenues pour certaines sépultures, confirmées parfois par une double datation (par ultrafiltration) sur ossements humains, qui seraient à rejeter sous le prétexte de l'existence d'une niche caractéristique du rituel funéraire du Néolithique ancien ou moyen et de la présence d'armatures en silex! Les recoupements évidents de ces sépultures avec des fosses latérales de construction et le type d'inhumation prônent pour l'existence d'une nécropole protohistorique. Certains faits, comme l'existence de structures polylobées, de recoupements, d'orientation divergente des unités, ou le maillage hyper serré de la rangée d'habitation sont simplement notés par les auteurs, parfois discutés, mais jamais retenus pour envisager d'autres hypothèses alternatives. 
À plusieurs reprises, les faits ou les résultats apparaissent discordants avec une attribution globale au RRBP, mais in fine n'inquiètent guère les auteurs : céramique VSG, anneaux en schiste, importance des pièces esquillées et des burins etc. Malheureusement ces " œillères " méthodologiques ont empêché de mettre en exergue toute la richesse et la dynamique chrono-culturelle de ce site clé, témoignant entre autres des débuts de la néolithisation du territoire normand à la fin du VIe millénaire, avec des composantes rubanées mais aussi Villeneuve-Saint-Germain, témoins d'un syncrétisme culturel.

Anne Hauzeur

Université de Liège - Paléotime

Thuillier F., Louis E. (dir.), 2015 - Tourner autour du pot. Les ateliers de potiers médiévaux de Vo au XIt siècle dans l'espace européen, Caen, PUC, coll. «Publications du Craham; série antique médiévale », 789 p.

Cet ouvrage est le résultat des actes du colloque international qui a eu lieu à Douai du 5 au 8 octobre 2010 et qui a eu pour thème les ateliers de potiers du premier Moyen Âge. Le choix de ce thème est lié au constat de lacunes existantes dans les études et les publications sur les fours de potiers postérieur au $\mathrm{IV}^{\mathrm{e}}$ siècle alors que la multiplications des découvertes à l'échelle européenne par les fouilles préventives permettaient grâces aux nouvelles données de mener une réflexion général et détaillée sur les différents aspects de la production céramique.

Cet ouvrage est introduit par 3 préfaces, un avant-propos et deux introductions. Celle de F. Verhaeghe porte sur les ateliers de potiers du haut Moyen Âge : acquis et questions. Il souligne l'objectif de faire un état de nos connaissances et de mettre l'accent sur les nouvelles données par rapport à la production matérielle que ce soit les fours, la céramique mais aussi les matières premières. Des questionnements sur les techniques et technologies sont posés ainsi que sur l'évolution chronologique et typo-chronologique des produits et des fours et parfois des ateliers. Plusieurs thèmes ont été soumis par les organisateurs : l'implantation, la répartition des fours et des ateliers ainsi que les caractéristiques de ceuxci ainsi que des structures des contextes de production. La diffusion des productions est également regardée. A. Wilkins s'attache à un bilan synthétique et aux axes de recherche sur les échanges artisanaux de haut Moyen Âge

La première partie présente les ateliers de potiers en France. Il s'agit essentiellement d'actualiser le corpus d'ateliers découverts en présentant à la fois les structures rencontrées et particulièrement les fours ainsi qu'une description des différentes productions de ces ateliers. Le constat d'un renouveau des données par l'apport notamment de l'archéologie préventive est évident au regard du grand nombre de contribution (21 articles). Les résultats présentés sont cepen- dant hétérogènes. Ils dépendent par exemple des différentes méthodes appliquées ou de la qualité des sites étudiés (superficie, importance de l'officine...). Trois régions présentent un état des lieux avec un recensement des ateliers découverts (Douaisie, Île-de-France, Centre). Deux centres de production importants connus depuis longtemps par l'archéologie renouvellent les données avec l'apport des nouvelles fouilles (La Saulsotte et Sevray).

Une deuxième partie se concentre sur le reste de l'espace européen. Il s'agit de 9 articles qui concernent 6 pays (Belgique, Bulgarie, Roumanie, Grèce, Espagne et Ouzbékistan) témoignant de l'état de la recherche. Ces études sur des espaces plus ou moins élargis sont importantes pour permettre des comparaisons.

Une troisième partie s'appuie sur l'aspect économique de l'artisanat potier en s'appuyant sur les données céramiques. La production, les faciès culturels et les échanges sont ainsi abordés par différents acteurs de l'archéologie européenne (France, Royaume Uni, Pays-Bas, Allemagne). Les données typologiques technologiques ainsi que les données macroscopiques (définition de groupe technique et de fabrics) et microscopiques (pétrographie, analyses chimiques) sont ainsi utilisés afin de souligner les changements tant culturel, chronologique et économique durant le premier Moyen Âge.

Enfin une dernière partie intitulée méthodologie, typologie et expérimentation se concentre sur les aspects technologiques et particulièrement des fours. Ainsi F. Thuilier présente un outil important de la recherche avec une nouvelle classification des fours de potier $\mathrm{du} \mathrm{v}^{\mathrm{e}}$ au XII ${ }^{\mathrm{e}}$ siècle en France, qui s'appuie sur le corpus d'atelier de potier médiévaux qui se veut exhaustif. Ce travail est mis en perspective grâce à la synthèse présenté par $\mathrm{A}$. Heege pour l'Allemagne, la Suisse, la Belgique, les Pays-Bas et l'Autriche. De mêmes les aspects de la source des matières premières, de la prépa- 